\title{
Avaliação da composição corporal em gestantes de termo
}

\author{
A.A.C. Morais, G.M. Tavares, A.C. Pezzin, A.A. Moana, H.P. Galvão, J . Faintuch \\ Escola de Medicina da Santa Casa de Misericórdia de Vitória, Vitória, ES.
}

\begin{abstract}
RESUMO - A gravidez acompanha-se de modificações fisiológicas bem conhecidas nos compartimentos hídricos e energéticos maternos, que se somam a hipertrofias orgânicas e ao aparecimento de um bloco feto-placentário. Conseqüentemente, a composição corporal sofre alterações, porém poucos estudos têm documentado tal fenômeno. $O$ advento da bioimpedância favoreceu a obtenção das informações desejadas de forma prática e segura.

Овл етіvos. Foram os propósitos deste trabalho: registrar as principais variáveis antropométricas numa população de gestantes de termo; determinar as medidas de composição corpórea pela bioimpedância; comparar esses achados com os primeiros, assim como com outras metodologias relatadas na literatura.

Pacientes e Método. A população consistiu de 30 gestantes de termo admitidas no período pré-parto, sem complicações, sofrimento fetal ou gravidez múltipla, e submetidas às seguintes mensurações: Antropometria - Peso, altura, índice de massa corporal, prega cutânea do tríceps, circunferência muscular do braço; Bioimpedância - Gordura corporal, massa magra, água total, água intra e extracelular, terceiro espaço e relação sódio/potássio permutável.
\end{abstract}

\section{INTRODUÇÃO}

O período final de gravidez acompanha-se de al terações fisiológicas nos compartimentos hídricos do organi $\mathrm{smo}^{1}$, ao lado de eventuais déficits e excessos nutricionai $\mathrm{s}^{2-4}$. Surge um conjunto feto-placentário significativo, cujo conteú do fluido se distingue dos tecidos maternos ${ }^{5}$, assim como hipertrofia de mamas e útero, tudo isto refletindo-se em medidas nutricionais e da composição corpórea.

Todavia, embora as curvas de peso na gravidez estejam amplamente documentadas ${ }^{6}$, poucos estudos aferiram as gestantes sob o prisma da composição corporal. Tendo-se em vista a facilidade e segurança da realização de tal propedêutica com o advento da bioimpedância, realizou-se um estudo sistemático desta natureza em gestantes de termo.

F oram objetivos do presente trabalho: 1) determinar a composi ção cor pórea em gestantes admitidas em trabalho de parto no final do terceiro
Resultados. Observaram-se valores de peso corporal e índice de massa corporal aumentados, porém inteiramente compatíveis com a situação fisiológica das gestantes. A água corpórea total situava-se na mesma faixa percentual de mulheres nãográvidas, tal como já suspeitado por outros autores, porém com indícios de expansão do extracelular e formação de terceiro espaço. A massa gorda elevou-se, porém, também em proporções não muito diferentes do relatado anteriormente em levantamentos antropométricos.

Conclusões. Nas condições do presente trabalho, em que uma equação de bioimpedância para uso geral foi aplicada, o método demonstrou resultados compatíveis com as informações clínicas disponíveis, as verificações antropométricas e os registros de outros investigadores. Conclui-se que a impedância bioelétrica é um procedimento de avaliação promissor na gravidez, justificando estudos ulteriores com esta metodologia.

UNITERMOS: Gravidez de termo. I mpedância bioelétrica. Composição corporal. Água corporal. Gordura corporal. Antropometria. Avaliação nutricional .

trimestre de gravidez; 2) comparar o perfil antropométri co e as mensurações de bi oi mpedância nesta população; 3) analisar esses mesmos achados à luz dos valores previstos pela evolução clínica e por estudos da literatura, no tocante às informações de compartimentos energéticos (massa magra, gordura corporal) e também de espaços hídricos (água corpórea total e suas subdivisões).

\section{PACIENTES E MÉ TODO}

A população consistiu em gestantes de termo, admitidas consecutivamente em trabalho de parto, e a avaliação da composição corpórea foi inserida na rotina de exame clínico pré-parto, quando atendidas as seguintes normas gerais:

Critérios de inclusão - I dade de 15-50 anos, primíparas ou multíparas; gestação tópica de termo ( $\geq 37$ semanas); consentimento ao exame. 
Critérios de exclusão - Gravidez múltipla, patológica ou sofrimento fetal; obesidade ou caquexia à avaliação antropométrica; insufi ciências orgânicas, sépsis ou choque; trabal ho de parto expulsivo.

Foram observadas, concomitantemente, as exigências habituais para exames de bioimpedância, a saber, jejum de pelo menos duas horas, com ou sem soro venoso de hidratação, e micção há menos de 30 minutos. Pacientes em anasarca ou uso recente de diuréticos, bem como aquelas clinicamente desidratadas ou com relato de vômitos ou diarréia, foram igualmente afastadas da avaliação-11.

O aparelho de bioimpedância utilizado (RJ L Systems, Clinton Township, MI, EUA) utiliza a técnica clássica tetrapolar, e uma freqüência fixa de $50 \mathrm{mHz}$. As gestantes eram posicionadas em decúbito dorsal horizontal, com os braços e pernas afastados do tronco, e as medidas obtidas automaticamente, com auxílio de quatro el etrodos posicionados dois a dois, respectivamente, no dorso da mão e do pé10-11. A equação do aparelho baseia-se nas medições de Lukaski \& Bol onchuk em populações sadias ${ }^{10}$.

As seguintes mensurações foram realizadas em todos os casos $(n=30)$ :

Avaliação antropométrica - Peso, altura, índice de massa corporal (IMC), prega cutânea do tríceps (PCT) e circunferência muscular do braço (CMB);

E stimati vas por bi oi mpedância - Gordura corporal, massa magra, água total, água extra e intracelular (AEC, AIC), terceiro espaço, e relação sódio/potássio permutável. As leituras de terceiro espaço foram interpretadas como edema (água fora do intra e do extracelular habituais), e a relação sódio/potássio permutável $\left(\mathrm{Na}_{\mathrm{e}} / \mathrm{K}_{\mathrm{e}}\right)$, como indicativa da intensidade da hipervolemia e expansão do extracelular, dada a distribuição preferencial do íon sódio fora das células e do potássio no citoplasma cel ular.

Os resultados absolutos ou percentuais foram expressos como média \pm desvio padrão. Para as determinações antropométricas, adotaram-se os critérios de normalidade de Bistrian ${ }^{7}$, sempre que aplicáveis, combinados com as observações de Olson et al. . $\mathrm{O}$ emprego da expressão "peso ideal" refere-se sempre ao peso de uma população de igual sexo, idade e altura, não-grávida, tal como fornecido por tabelas convencionais de relação peso/altura. Consideraram-se como sobrepeso e obesidade aumentos médios de, respectivamente, menos de $20 \%$ e mais de $20 \%$ nas determinações antropométricas, e desnutrição leve ou grave (caquexia), diminuições de também menos de $20 \%$ e mais de $20 \%$ nas mesmas medidas.
Tabela 1 - Informações gerais e antropométricas

$\begin{array}{ll}\text { Idade (anos) } & 26,6 \pm 7,4 \\ \text { Peso (kg) } & 72,0 \pm 12,1 \\ \text { Prega cutânea do tríceps (mm) } & 16,7 \pm 7,3 \\ \text { Prega cutânea do tríceps (desvio percentual) } & +1,5 \pm 44,1 \\ \text { Circunferência muscular do braço (cm) } & 22,6 \pm 3,6 \\ \text { Circunferência muscular do braço (desvio percentual) } & -2,4 \pm 15,3 \\ \text { Índice de massa corporal }\left(\mathrm{kg} / \mathrm{m}^{2}\right) & 28,6 \pm 3,9\end{array}$

Tabela 2 - Resultados da bioimpedância

Massa magra $(\mathrm{kg})$

Massa magra (\%)

Gordura corporal $(\mathrm{kg})$

Gordura corporal (\%)

Água corpórea total (L)

Água corpórea total (\%)

Água intracelular (L)

Água intracelular (\%)

Água extracelular (L)

Água extracelular (\%)

$3^{\circ}$ espaço $(L)$

3ำ espaço (\%)

Relação $\mathrm{Na} / \mathrm{K}$ permutável $\left(\mathrm{Na}_{\mathrm{e}} / \mathrm{K}_{\mathrm{e}}\right)$

$49,6 \pm 8,3$

$69,2 \pm 5,1$

$22,3 \pm 5,9$

$30,8 \pm 5,1$

$35,9 \pm 6,1$

$50,0 \pm 3,7$

$18,6 \pm 2,1$

$52,3 \pm 4,8$

$15,9 \pm 2,3$

$44,5 \pm 1,5$

$1,7 \pm 2,3$

$4,0 \pm 4,8$

$0,95 \pm 0,09$

\section{RESULTADOS}

Antropometria - As gestantes exibiram um excesso de peso de 29,9 $\pm 17,7 \%$, compatível com um diagnóstico nominal de obesidade, o mesmo sucedendo com o IMC $\left(28,6 \pm 3,9 \mathrm{~kg} / \mathrm{m}^{2}\right)^{7,8}$. Seu peso ideal, cal culado para uma população idêntica fora de gravidez, foi de 55,4 $\pm 9,3 \mathrm{~kg}$. O ganho de peso estimado por conta da gravidez foi, portanto, de $16,6 \mathrm{~kg}$, em média, para um valor desejável de $10-15 \mathrm{~kg}^{6,8}$.

As determinações de PCT e CMB estiveram muito próximas do ideal. Seus desvi os padrões um pouco alargados rel acionam-se à incl usão no estudo de casos com pequenos desvios nutricionais, fora da faixa de obesidade e desnutrição grave (tabela 1).

Impedância elétrica - Registrou-se um déficit relativo de massa magra à luz dos padrões convencionais (valor medido 69,2 $\pm 5,1 \%$, normal $74-80 \%$ ), associado a excesso equival ente de gordura corporal $(30,8 \pm 5,1 \%$, normal $20-26 \%)$. Esses desvios não foram confirmados pela antropometria. No tocante à água corpórea total (normal 49-57\%), AIC (52$61 \%$ ) e AEC (39-48\%), os achados situaram-se na faixa aceitável ou próximos ao limite inferior do normal. Havia um terceiro espaço bem marcado $(4,0$ $\pm 4,8 \%$ ) e uma relação $\mathrm{Na}_{e} / \mathrm{K}_{\mathrm{e}}$ próxima ao limite superior (normal até 0,98), compatível com uma sobrecarga hídrica extracelular (tabela 2). 


\section{DISCUSSÃO}

A impedância bioelétrica representa um dos primeiros processos rápidos, não-invasivos, não ionizantes, e de confiabilidade aceitável para a quantificação dos compartimentos corporais, principalmente os de natureza fluida. Sua origem fundamenta-se em observações de há mais de três décadas, que evidenciavam diferenças nas propriedades elétricas dos distintos tecidos do organismo, e as relacionavam a seu conteúdo de água e el etrólitos ${ }^{9}$. Tornou-se claro que com a documentação da resistência (impedância) e reactância corporal, e sua interpretação por meio de equações adequadas, brevemente se poderia, mediante um único teste, fornecer o perfil dos principais componentes energéticos e hídricos do organismo, tal como hoje praticado.

A val idade dessa abordagem não é mais questionada em adultos metabolicamente estáveis e sem aber rações orgânicas significativas ${ }^{10,11}$. Sua aplicação em pediatria e neonatologia, bem como na obesidade e desnutrição, também se revela viável, embora demandando, algumas vezes, equações ajustadas para a população alvo ${ }^{5}$. As maiores restrições dizem respeito a pacientes com distúrbios acentuados dos espaços hídricos, tais como indivíduos com edemas e derrames cavitários ou enfermos críticos, quando a precisão do método poderá estar comprometida ${ }^{11,12}$.

As gestantes de termo sem disfunções orgânicas podem ser definidas como uma população essencialmente jovem e sadia, porém compartilhando algumas aberrações hídricas e compartimentais próprias de grupos enfermos. Nesse sentido, é lícito indagar se a pletismografia elétrica tradicional alcançaria sucesso neste contexto.

Relata-se que o aumento da volemia na gravidez, principalmente na sua fração plasmática, reduz o hematócrito, o que poderá interferir nas estimativas da bioimpedância. Também a geometria corporal sealtera, afetando uma das premissas do cál culo da resistência e reactância, que é o diâmetro do corpo atravessado pela corrente ${ }^{1}$. Outros enfatizam que o gl icogênio tecidual tende a se depletar no final da gestação, com concomitante perda de água celuIar, resultando em mudança da densidade da massa magra e fal has na estimação del $a^{13}$.

Diante de todas estas incertezas, Lukaski et al. ${ }^{1}$ optaram por estudar a água corporal na gravidez com base em equação própria, obti da por regressão linear múlti pla não somente das medidas usuais de resistência, peso e altura, mas levando em conta, também, a reactância, circunferência abdominal e hematócrito. Sua proposta é aqui alinhada com a equação padrão por nós utilizada:
Fórmula geral ${ }^{10}-$ Água total $=0,61$ (altura $2 /$ resistência) +0,063 (peso) +0,06.

Fórmula ajustada para gravidez (Lukaski et al. $\left.{ }^{1}\right)$ - Água total $=0,7$ (altura ${ }^{2} /$ resistência $)+^{2}$ 0,051 (circunferência abdominal) - 0,069 (peso) 0,029 (reactância) - 0,043 (hematócrito) + 2,833.

Validando ambas as alternativas com o padrãoouro da di luição isotópica com deutério, os autores citados concluíram que, para um ganho hídrico real (deutério) de 7,1 $\pm 1,1 \mathrm{~kg}$ ao longo de toda a gestação, a bioimpedância convencional ressentia-se de uma subavaliação para apenas $5,4 \pm 1,1 \mathrm{~kg}$. Lançando mão de sua equação aprimorada, essa deficiência corrigia-se em parte, atingindo-se um valor esti mado de 6,2 $\pm 1,0 \mathrm{~kg}$, mais condizente, portanto, com o acréscimo real.

Cabe frisar, entretanto, que em termos relativos, a fórmula de Lukaski et al. ${ }^{1}$ chegou a uma taxa de água na gravidez de $50,0 \pm 1,4 \%$, idêntica à nossa cifra de $50,0 \pm 3,7 \%$ (tabela 2 ). Podemos confiar, destarte, que, ainda que nossas medições não tenham recorrido às interessantes recomendações daquel es especialistas, mesmo assim el as merecem crédito, ao menos no que concerne aos espaços hídricos. Ressalte-se que a metodologia de Lukaski et al. ${ }^{1}$ não permitiu a dedução da AIC, $A E C$ eterceiro espaço, mas é plausível que também essas l eituras sejam um reflexo fidedigno da gestação de termo. Especificamente, a presença de um pequeno mas bem individualizado terceiro espaço (aproxi madamente 4\% da água corporal) se coaduna com a freqüente detecção clínica de um certo edema de tornozelo nesta população, do mesmo modo como a relação $\mathrm{Na}_{\mathrm{e}} / \mathrm{K}_{\mathrm{e}}$ no limite superior do normal é fortemente indicativa de hipervolemia e expansão do extracelular, o que coincide com os conhecimentos e a experiência prática.

Tal como sucedeu com algumas mensurações de compartimentos hídricos, não encontramos na literatura estudos equivalentes com emprego da bioimpedância que permitissem uma comparação direta com nossos resultados, no tocante à massa magra e à gordura corporal. Como mencionado no capítulo de resultados, à primeira vista, a el evação da gordura e conseqüente diminuição da massa magra não parecem encontrar respal do nos estudos antropométricos efetuados simultaneamente, visto que a prega trici pital e a circunferência muscular do braço encontravam-se quase coincidentes com a normalidade.

Nessas condições, recorremos aos estudos de Villar et al. ${ }^{14}$, em que a composição corporal na gestação é antecipada com apoio em determinações antropométricas. As mensurações diretas dos autores direcionam-se à $10^{a}$ semana de evolução, 
mas, à luz das curvas de aumento corporal projetadas para todo o ciclo gravídico, pôde-se chegar, facilmente, aos valores esperados a termo.

Uma vez computados os índices de IMC de $26,7 \mathrm{~kg} / \mathrm{m}^{2}$, massa magra de $67,4 \%$ e gordura corporal equivalente a $34,8 \%$, houve condições de comparação com os estudos aqui trazidos. Percebemos nas tabelas 1 e 2 que nossas gestantes atingiram IMC de $28,6 \pm 3,9 \%$, gordura total de $30,8 \pm 5,1 \%$ e massa magra de $69,2 \pm 5,1 \%$, nitidamente aberrantes quando cotejados com os parâmetros normais, ou mesmo com as leituras de antropometria realizadas concomitantemente, mas bastante próximas do correto para gestantes de termo segundo Villar et al. ${ }^{14}$

É legítimo afirmar-se, nessas condições, que, mesmo tendo aplicado equação de utilização geral sem correções próprias para gestantes, as estimativas da bioimpedância se revelaram precisas para a água corpórea total, e pouco desviadas do esperado para os demais compartimentos hídricos e energéticos. A busca de equações aperfeiçoadas é inteiramente lógica e deverá ser nosso próximo passo. De qualquer modo, foi encorajador constatar que, mesmo na ausência de ajustes específicos, a avaliação da composição corporal por bioimpedância já se demonstrou capaz de contribuir para o estudo e a documentação das al terações fisiológicas, neste importante grupo populacional.

Nossos achados sobre teor hídrico das grávidas merecem um reforço final. Há consenso geral de que a gravidez deve ser entendida como uma extensa soma al gébrica, em que o principal acúmulo molecular se refere à água, porém é polêmico se a participação desta é da ordem de $50 \%, 60 \%$ ou mesmo $70 \%{ }^{1}$.

As incertezas são menores quando o ganho de peso é decomposto entre grandes compartimentos energéticos, atribuindo-se parcelas pouco desiguais à gordura (54\%) e à massa magra $(46 \%)^{14}$. As operações matemáticas se alongam novamente quando nos recordamos que, nos totais anteriores, os tecidos fetais participam com cerca de $40 \%$ do peso ${ }^{14}$, porém propor ções teci duais absol utamente diversas: o neonato é formado em mais de $80 \%$ de água ${ }^{5}$, com frações rel ativamente modestas de proteína e panículo adiposo, e no âmbito placentário, novamente, avulta o líquido amniótico.

Nossos testes de bioimpedância insistem em que a água corporal na gestação de termo situouse em $50 \%$ do peso (tabela 2 ), um valor suspeito por ser quase coinci dente com o de mulheres nãográvidas. Não estaria ocorrendo, aqui, uma falha do método, uma insensibilidade da pl etismografia elétrica aos importantes remanejamentos inter- nos secundários ao ci cl o reprodutivo? Há ponderáveis motivos para se afirmar que não. Os excessos hídricos do feto são reais, mas a contrapartida é o ganho de gordura ( $90 \%$ materna $\left.{ }^{14}\right)$, quase anidra, resultando numa parcela aquosa média de $50 \%$, anál oga à dos teci dos maternos pré-gravídicos, de sorte a, praticamente, não modificar as leituras de bioimpedância. Tal suposição encontra respaldo nos resultados da equação de Lukaski et al. ${ }^{1}$, assim como, com poucas ressalvas, de outros grupos experientes ${ }^{13}$.

\section{CONCLUSÕES}

Numa investigação de 30 gestantes de termo consecutivamente admitidas no período pré-parto, e avaliadas do ponto de vista da antropometria e da bi oimpedância, as di mensões dos distintos compartimentos corporais - embora compreensivelmente distorcidas sob o prisma de uma população fora da gravidez - denotaram concordância bastante aceitável com os valores esperados à luz da fisiologia da gestação, dos demais achados clínicos e dos relatos da literatura.

Conclui-se que a impedância bioelétrica parece ser dotada de potencial para detecção dos desvios hídricos e energéti cos, justificando-se o prosseguimento das investigações nesta área.

\section{SUMMARY}

\section{Assessment of body composition in pregnant women at term}

BACKGRound. Pregnancy is associated with wellknown physiologic changes of maternal fluid and energy compartments, along with organ hypertrophies and the appearance of fetal and placental tissues. As a consequence, body composition is modified, but this phenomenon has not been well documented. The advent of bi oi mpedance has contributed to the documentation of the desired information in a safe and practical way.

Aıms. Theaims of this study were: To register the principal anthropometric variables in a population of pregnant women at term; To determine body composition by bi oi mpedance analysis; To compare these findings with the former results, as well as with other assessment procedures reported in the literature.

Patients and Method. The population consisted of 30 pregnant women at term admitted for delivery, without complications, fetal distress or multiple pregnancy, and submitted to the following measurements: Anthropometry - Weight, height, 
body mass index, triceps skinfold, arm muscle circumference; Bioimpedance analysis - Body fat, lean body mass, total water, intra and extracellular water, third space, and exchangeable $\mathrm{Na} / \mathrm{K}$ ratio.

RESULTS. Body weight and body mass index were increased but within the expected values for these pati ents. Total body water was similar to results in non-pregnant women when expressed as percentage, in accordance with other studies, but with a trend toward increase in the extracellular compartment and presence of third space fluid. Body fat was el evated, but the proportions were not much different from previous anthropometric surveys.

ConCLUSIONS. In the conditions of this investigation, in which a bioimpedance equation for general use was employed, the method indicated results that were consistent with the clinical course, anthropometric documentation, and the findings of other groups. It is concluded that bioimpedance analysis compares favorably with other assessment procedures in pregnancy, and further studies with this method should be undertaken. [Rev Ass Med Brasil 1997; 43(2): 109-13.]

KEY WORDS: Pregnancy at term. Bioelectric impedance analysis. Body composition. Body water. Body fat. Anthropometry. Nutritional assessment.

\section{REFERÊNCIAS BIBLIOGRÁFICAS}

1. Lukaski HC, Siders WA, Nielsen EJ, Hall CB. Total body water in pregnancy: assessment by using bioelectrical impedance. Am J Clin Nutr 1994; 59: 578-85.

2. Carriaga MT, Skikne BS, Finley B, Cutler B, Cook J D. Serum transferrin receptor for the detection of iron deficiency in pregnancy. Am J Clin Nutr 1991; 54: 1.077-81.
3. Barnard HC, De Kock JJ, Vermaak WJ H, Potgieter GM. A new perspective in the assessment of vitamin B-6 nutritional status during pregnancy in humans. J Nutr 1987; 117: 1.303-6.

4. Perlow J H, Morgan MA, Montgomery D, Towers CV, Porto M. Perinatal outcome in pregnancy complicated by massive obesity. Am J Obstet Gynecol 1992; 167: 958-62.

5. Mayfield SR, Vaauy R, Waidelich D. Body composition of low-birth-weight infants determined by using bioelectrical resistance and reactance. Am J Clin Nutr 1991; 54: 296-303.

6. Institute of Medicine: Nutrition during pregnancy, weight gain and nutrient supplements: report of the Subcommittee on Nutritional status and weight gain during pregnancy, Subcmmittee on Dietary intake and nutrient supplements during pregnancy, Committee on Nutritional status during pregnancy and lactation, Food and Nutriton Board. Washington DC, USA, National Academy Press, 1990.

7. Bistrian BR. Anthropometric norm used in assessment of hospitalized patients. Am J Clin Nutr 1980; 33: 2.211-4.

8. Olson C, Wilson J , Pitkin RM Nutrition during pregnancy and Iactation: an implementation guide National Academy Press, Washington, DC, USA, 1992.

9. Nyboer J . Workable volume and flow concepts of bio-segments by electrical impedance plethysmography. T-I-T-J Life Sci 1972; 2: 1-13.

10. Lukaski Hc, Bolonchuk WW. Theory and validation of the tetrapolar bioelectrical impedance method to assess human composition. In Ellis KJ, Yasamura S, Morgan WD (eds): Proceedings of na international symposium of in-vivo body composition studies. The Institute of Medicine and Physics, London, 1987; 410-4.

11. Holt TL, Cui C, Thomas BJ et al. Clinical applicability of bioelectric impedance to measure body composition in health and disease. Nutrition 1994; 10: 221-4.

12. Chumlea WC, Guo SS. Bioelectrical impedance and body composition: present status and future directions. Nutr Ver 1994; 52: 123-31.

13. Van Raaij J MA, Peek MEM Vermaat-Miedema SH et al. New equations for estimating body fat mass in pregnancy from body density or total body water. Am J Clin Nutr 1988; 48: 24-9.

14. Villar J , Cogswell M, Kestler E et al. Effect of fat and fat-free mass deposition during pregnancy on birth weight. Am J Obstet Gynecol 1992; 167: 1.344-52. 